\title{
Fluorescence immunoassay through histone-ds-poly(AT)-templated copper nanoparticles as signal transductors for the sensitive detection of Salmonella choleraesuis in milk
}

\author{
Ying Xiong, ${ }^{1,2}$ Kangkang Zhang, ${ }^{1}$ Bao Gao, ${ }^{1,2}$ Yunqing Wu, ${ }^{1,2}$ Xiaolin Huang, ${ }^{1}$ Weihua Lai, ${ }^{1}$ Yonghua Xiong, ${ }^{1,2 *}$ \\ and Yang Liu,4* \\ ${ }^{1}$ State Key Laboratory of Food Science and Technology, Nanchang University, Nanchang 330047, P. R. China \\ ${ }^{2}$ Jiangxi Sino-German Joint Research Institute, Nanchang University, Nanchang 330047, P. R. China \\ ${ }^{3}$ Institute of Food Science and Technology, Chinese Academy of Agricultural Sciences, Beijing 100193, P. R. China \\ ${ }^{4}$ Key Laboratory of Agro-products Quality and Safety Control in Storage and Transport Process, Ministry of Agriculture and Rural Affairs, \\ Beijing 100193, P. R. China
}

\section{ABSTRACT}

The rapid and sensitive detection of foodborne pathogens is one of the most important issues in food safety control. In this work, we developed a novel fluorescence immunoassay method for the sensitive detection of Salmonella choleraesuis. The method uses the fluorescent signals of histone-ds-poly(AT)-templated copper nanoparticles (His-pAT CuNP) as signal transducers and glucose oxidase as an alternative for horseradish peroxidase for the generation of hydrogen peroxide $\left(\mathrm{H}_{2} \mathrm{O}_{2}\right)$ through the catalysis of glucose. The $\mathrm{H}_{2} \mathrm{O}_{2}$ is then further converted into hydroxyl radical $(\cdot \mathrm{OH})$ by Fenton reagents. Owing to the ultrahigh sensitivity of His-pAT CuNP synthesis toward $\cdot \mathrm{OH}$, the proposed fluorescence immunoassay method exhibited excellent sensitivity for $S$. choleraesuis, with a limit of detection of $8.04 \times 10^{1} \mathrm{cfu} / \mathrm{mL}$, which is 3 orders of magnitude lower than that of the tetramethylbenzidine-based traditional immunoassay. The reliability of the proposed method was evaluated by using spiked milk samples with $S$. choleraesuis concentration ranging from $8.8 \times$ $10^{1}$ to $8.8 \times 10^{4} \mathrm{cfu} / \mathrm{mL}$. The average recoveries for the intra- and inter-assay ranged from 73.52 to $96.59 \%$ and from 66.99 to $98.24 \%$ with a coefficient of variation from 6.85 to $31.26 \%$ and 5.46 to $17.99 \%$, respectively. These results indicated that the proposed fluorescence immunoassay possesses a great potential for ultra-sensitive detection of foodborne pathogens in food safety control.

Key words: Salmonella choleraesuis, fluorescence immunoassay, DNA-templated copper nanoparticle, milk

\footnotetext{
Received February 12, 2019.

Accepted March 19, 2019.

*Corresponding authors: yhxiongchen@163.com and liuyang01@ caas.cn
}

\section{INTRODUCTION}

Foodborne pathogens pose important public health concern worldwide because they can contaminate foods through any food chains from farm to fork (Shan et al., 2015; Shang et al., 2018). The Centers for Disease Control and Prevention estimated that 1 in 6 Americans (or 48 million people) each year becomes adversely affected by foodborne diseases (CDC, 2018). The World Health Organization estimated that approximately a million people suffer from foodborne disease each year in the United Kingdom (WHO, 2011). The traditional culture-based approach used for foodborne pathogen detection usually takes up 5 to $6 \mathrm{~d}$ to obtain determined results, failing to meet the requirement of rapid screening detection (Abdalhai et al., 2014, 2015). Various simple, specific, and rapid methods, such as real-time multiplex PCR (Hiitiö et al., 2018), immunochromatographic strips (Luo et al., 2018), and ELISA have been developed (Chen et al., 2016). Among them, ELISA is one of the most popular methods for high-throughput screening detection of foodborne pathogen-polluted samples. However, the sensitivity of traditional ELISA for foodborne pathogen detection employing horseradish peroxidase (HRP) to generate colored molecules as signal outputs usually ranges from $10^{4}$ to $10^{6} \mathrm{cfu} /$ $\mathrm{mL}$ (Chen et al., 2015). Thus, a highly sensitive ELISA method for detecting low level of foodborne pathogens is still required.

In recent years, methods for synthesizing DNA-templated fluorescent metal nanoparticles, such as gold and silver nanoclusters and copper nanoparticles (CuNP; Petty et al., 2004; Chakraborty et al., 2015; Mao et al., 2015; Lian et al., 2017), have been developed. These novel DNA-templated fluorescent nanoclusters or nanoparticles show great potential as signal reporters because of their excellent biocompatibility and superior fluorescence properties (Liu, 2014). Among them, 
DNA-templated CuNP have distinct advantages, such as instantaneous reaction, low toxicity and price, and large Stokes shifting (>200 nm; Qing et al., 2013). Long ss-DNA templates can be easily cleaved into monooligonucleotides or short oligonucleotides by the $\cdot \mathrm{OH}$, thereby reducing fluorescence signal because the synthesis of DNA-templated CuNP is damaged (Tang et al., 2006). Thus, DNA-templated CuNP are ideal signal transducers for fabricating $\cdot \mathrm{OH}$-dependent biosensors. To our best knowledge, fluorescence immunoassay by using $\cdot \mathrm{OH}$ to regulate the synthesis of DNA-templated CuNP as a signal transducer for the ultrasensitive detection of foodborne pathogens has not been reported.

In the present study, the histone-ds-poly(AT) templated (His-pAT) CuNP were ingeniously integrated into a sandwich immunoassay platform as fluorescent signal reporter. Salmonella choleraesuis was selected as the model target because it is an important nontyphoidal Salmonella bacterium and narrowly infects humans, causing diseases in all ages. Glucose oxidase (Gox) was used as the substitute of HRP for hydrogen peroxide $\left(\mathrm{H}_{2} \mathrm{O}_{2}\right)$ generation through glucose oxidation, and $\mathrm{H}_{2} \mathrm{O}_{2}$ was then converted into $\cdot \mathrm{OH}$ by Fenton reagent. Owing to the high sensitivity of His-pAT CuNP growth toward $\cdot \mathrm{OH}$, the limit of detection (LOD) of the proposed method reached $8.04 \times 10^{1} \mathrm{cfu} / \mathrm{mL}$, which is approximately 1,000-fold lower than that of tetramethylbenzidine (TMB)-based conventional immunoassay $\left(8.7 \times 10^{4} \mathrm{cfu} / \mathrm{mL}\right)$. The detection performances of the developed method were evaluated on the basis of specificity, precision, and practicability by using other common foodborne pathogens and $S$. choleraesuisspiked milk samples. The results demonstrated that the proposed fluorescence immunoassay showed great potential for sensitive detection of $S$. choleraesuis in real milk samples.

\section{MATERIALS AND METHODS}

\section{Materials and Apparatuses}

Magnetic beads (180 nm) were purchased from Allrunnano Technology Co. Ltd. (Shanghai, China). NHydroxysuccinimide sodium salt activated long-chain biotin (bio) and streptavidin (SA) were purchased from Hualan Technology Co. Ltd. (Shanghai, China). Copper sulfate pentahydrate, iron dichloride tetrahydrate, ascorbic acid, histone (from calf thymus, type III-S), 3 -( $N$-morpholino)-propanesulfonic acid (MOPS), TMB, BSA, HRP, Gox, and glucose were purchased from Sigma-Aldrich Chemical Co. (St. Louis, MO). The designed ss-poly(AT)-1 (5'-AATAATAATCTATAATAATT-3') and ss-poly(AT)-2 (5'-AATTATTATA-
GATTATTATT-3') were commercially synthesized by Sangon Biological Engineering Technology and Service Co. Ltd. (Shanghai, China). The electron microscopy supplies were purchased from Beijing Zhongjinkeyi Technology Co. Ltd. (Beijing, China). A pair of monoclonal antibodies against $S$. choleraesuis, labeled as 11D8-D4 and 5F11-B11, were used as the detection antibodies $(\mathbf{d A} \mathbf{b})$ and the capture antibodies $(\mathbf{c A b})$, respectively. Pasteurized milk was purchased from the local supermarket. The other reagents used were of analytical grade and purchased from Sinopharm Chemical Corp. (Shanghai, China). Chemicals and materials were employed without further purification. Ultrapure water was obtained from a Milli-QA apparatus (Molsheim, France). The 96-well plates (high binding and white or black) were obtained from Corning Inc. (Corning, NY). The $\mathrm{H}_{2} \mathrm{O}_{2}$ kit was purchased from Beijing ComWin Biotech Co. Ltd. (Beijing, China). Fluorescence signals were measured on Varioskan LUX (Thermo Fisher, Vantaa, Finland). Transmission electron microscopy analyses were performed on a JEM-2100 electron microscope operating at $100 \mathrm{kV}$ (Tokyo, Japan).

\section{Bacteria and Culture Conditions}

Salmonella choleraesuis (ATCC 10708), S. enteritidis (ATCC 13076), and other common pathogenic bacterial strains, including Escherichia coli O157:H7 (ATCC 43888), Listeria monocytogenes (ATCC 13932), Listeria welshimeri (ATCC 35897), Enterobacter sakazakii (CMCC 45401), Vibrio parahaemolyticus (PVPA 0146), Proteus vulgaris (CMCC 49027), and Bacillus subtilis (collection), were grown in Luria-Bertani medium overnight at $37^{\circ} \mathrm{C}$. The harvested bacterial solutions were continuously diluted with sterile PBS to different concentrations for the following experiments. The cell number of bacteria was determined by counting the colonies grown on the plates.

\section{Synthesis of His-pAT CuNP}

The CuNP were synthesized according to our previously described method (Xiong et al., 2018). First, the ss-poly(AT)-1 and ss-poly(AT)-2 were annealed at $90^{\circ} \mathrm{C}$ in MOPS buffer $(10 \mathrm{mM}$ MOPS and $150 \mathrm{mM}$ $\mathrm{NaCl}$ at $\mathrm{pH}$ 7.6) for 10 min, then quickly cooled down to room temperature $\left(25^{\circ} \mathrm{C}\right)$. Second, both $0.63 \mu M$ of ss-poly(AT)-1 and ss-poly(AT)-2 were mixed for the assembly of ds-poly(AT). After the solution was mixed for $1 \mathrm{~min}, 10 \mathrm{mg} / \mathrm{mL}$ of histone was added into solution for another $1 \mathrm{~min}$ for the reaction to form histone-dspoly(AT) complex, which is a template for CuNP synthesis. Finally, $5 \mathrm{mM}$ ascorbic acid and $2.5 \mathrm{mM} \mathrm{Cu} \mathrm{Cu}^{2+}$ 
were sequentially added. In a typical run, the added volume of ss-poly(AT)-1, ss-poly(AT)-2, ascorbic acid, and $\mathrm{Cu}^{2+}$ were $20 \mu \mathrm{L}$ individually, and the histone was $40 \mu \mathrm{L}$.

\section{His-pAT CuNP-Based Fluorescence Immunoassay for S. choleraesuis Detection}

The 96-well microplates were coated with $100 \mu \mathrm{L}$ of cAb $(2.5 \mu \mathrm{g} / \mathrm{mL})$ in bicarbonate buffer $(0.01 M$ at $\mathrm{pH}$ $8.6)$ at $4^{\circ} \mathrm{C}$ overnight and then blocked with $1 \mathrm{mg} / \mathrm{mL}$ of BSA solution at $37^{\circ} \mathrm{C}$ for $1 \mathrm{~h}$. After washing 3 times with PBS with Tween 20 (PBST), $100 \mu \mathrm{L}$ of the desired final concentrations of $S$. choleraesuis was added and incubated at $37^{\circ} \mathrm{C}$ for $1 \mathrm{~h}$. After washing 3 times with PBST, $100 \mu \mathrm{L}$ of biotinylated dAb (dAb@bio, 2.5 $\mu \mathrm{g} / \mathrm{mL}$ ) was added into the plates for $1 \mathrm{~h}$ at $37^{\circ} \mathrm{C}$. After washing the plates 3 times with PBST, $100 \mu \mathrm{L}$ of SA $(1.25 \mu \mathrm{g} / \mathrm{mL})$ was added. Following the 30-min reaction at $37^{\circ} \mathrm{C}$ and after washing the plates 3 times with PBST, $100 \mu \mathrm{L}$ of biotinylated Gox (Gox@bio; $10 \mu \mathrm{g} / \mathrm{mL}$ ) was added, and the plates were incubated for $45 \mathrm{~min}$ at $37^{\circ} \mathrm{C}$. After washing the plates 4 times with PBST and twice with ultrapure water, $140 \mu \mathrm{L}$ of the solution containing $0.5 \mathrm{M}$ of glucose, $0.63 \mu \mathrm{M}$ of ss-poly(AT)-1, and $0.5 \mathrm{~m} M$ of $\mathrm{Fe}^{2+}$ were added to each well. After reacting at $37^{\circ} \mathrm{C}$ for $60 \mathrm{~min}$ of the above solution, $20 \mu \mathrm{L}$ of the ss-poly(AT)-2 $(0.63 \mu M)$ was added to each well for 1 min hybridization, and $40 \mu \mathrm{L}$ of histone $(10 \mathrm{mg} / \mathrm{mL})$ was added for another $1 \mathrm{~min}$ reaction. Finally, $20 \mu \mathrm{L}$ of ascorbic acid $(5 \mathrm{mM})$ and $20 \mu \mathrm{L}$ of $\mathrm{Cu}^{2+}(2.5 \mathrm{mM})$ were sequentially added. The fluorescence intensity (FI) from the formed CuNP was determined (excitation and emission at 340 and 600 $\mathrm{nm}$, respectively) by using a microplate reader within 10 min. A TMB-based conventional immunoassay was developed for comparison with detailed procedure provided in the Supplemental Information (https://doi .org/10.3168/jds.2019-16472).

\section{Detection of S. choleraesuis in Spiked Milk Samples}

Pasteurized milk was spiked with $S$. choleraesuis at different concentrations and detected through the proposed method. Salmonella choleraesuis was isolated, and its concentration was determined according to our previously developed 2-step large-volume immunomagnetic separation method (Luo et al., 2017). In brief, 10 $\mathrm{mL}$ of milk was added into $90 \mathrm{~mL}$ of $0.01 \mathrm{M}$ sterilized PBS to form a $10 \%$ homogenate. The S. choleraesuis solutions were spiked to the above homogenates with $S$. choleraesuis to final concentrations ranging from $8.8 \times$ $10^{1}$ to $8.8 \times 10^{4} \mathrm{cfu} / \mathrm{mL}$. Approximately $3 \mu \mathrm{g}$ of dAb@ bios was added into $S$. choleraesuis-spiked homogenate milk. After the homogenized milk was incubated at $37^{\circ} \mathrm{C}$ for $1 \mathrm{~h}$ with gentle shaking, $700 \mu \mathrm{g}$ of SA-modified magnetic beads was added, and the mixture solution was incubated for another $1 \mathrm{~h}$ at $37^{\circ} \mathrm{C}$ with gentle shaking. Finally, the target of $S$. choleraesuis was separated from the mixture with an external magnetic field. The separated $S$. choleraesuis was dissociated from the magnetic beads by heating at $90^{\circ} \mathrm{C}$ for $15 \mathrm{~min}$ and resuspended in $1 \mathrm{~mL}$ of PBS. The supernatant containing S. choleraesuis was analyzed by the proposed method.

\section{RESULTS AND DISCUSSION}

\section{Principle of Fluorescence Immunoassay for S. choleraesuis Detection}

The detailed principle of the proposed fluorescence immunoassay is depicted in Figure 1. The anti- $S$. choleraesuis monoclonal antibody and biotinylated anti- $S$. choleraesuis monoclonal antibody were employed as the $\mathrm{cAb}$ and dAb, respectively. The Gox@bio was used as an alternative for the HRP enzyme. The SA-bio system was used as a bridge to connect dAb@bio and Gox@ bio. When S. choleraesuis was present in the sample solution, Gox was immobilized on the plate wells by the SA-bio system. Glucose was oxidized by Gox for $\mathrm{H}_{2} \mathrm{O}_{2}$ production, and $\mathrm{H}_{2} \mathrm{O}_{2}$ was converted into $\cdot \mathrm{OH}$ with Fenton reagent $\left(\mathrm{Fe}^{2+}\right)$. Then, ss-poly (AT)-1 was cleaved into mono-oligonucleotides or short oligonucleotides by . OH, resulting in a weak FI on the plate wells because of a failed ds-poly(AT) template for CuNP synthesis. Decrease in $\mathrm{H}_{2} \mathrm{O}_{2}$ generation increased His-pAT CuNP synthesis and high FI. The concentration of $S$. choleraesuis can be quantitatively determined by recording the fluorescence retention of His-pAT CuNP because $S$. choleraesuis concentration is inversely proportional to the amount of His-pAT CuNP.

\section{His-pAT CuNP-Based Fluorescent Signal Transducer}

The His-pAT CuNP were synthesized by using a ds-poly(AT) DNA as template, ascorbic acid was used for reducing $\mathrm{Cu}^{2+}$ to $\mathrm{CuNP}$, and histone was used for enhancing the FI and stability of the pAT CuNP. The optimum concentrations of ds-poly(AT), ascorbic acid, $\mathrm{Cu}^{2+}$, and histone for His-pAT-CuNP synthesis were based on our previous report (Xiong et al., 2018). Figure 2A shows that the as-prepared His-pAT CuNP showed a bright red fluorescence under UV lamp excitation at $365 \mathrm{~nm}$. In the fluorescence spectra of the His-pAT CuNP, the maximum excitation and emission wavelengths were 340 and $600 \mathrm{~nm}$, respectively (Figure 2B). The transmission electron microscopy images indi- 
cated that most of CuNP are approximately spherical with good mono-dispersity (Figure 2C).

The sensitivity of His-pAT CuNP growth to $\cdot \mathrm{OH}$ was evaluated by incubating ss-poly(AT)-1 with different concentrations of $\mathrm{H}_{2} \mathrm{O}_{2}(0-50 \mu M)$ in the presence of 0.5 $\mathrm{m} M \mathrm{Fe}^{2+}$. Fluorescence retention of resultant His-pAT $\mathrm{CuNP}$ was calculated with the formula $\mathrm{F} / \mathrm{F}_{0} \times 100 \%$, where $\mathrm{F}$ and $\mathrm{F}_{0}$ represent the fluorescence intensities of His-pAT CuNP solution with or without the desired $\mathrm{H}_{2} \mathrm{O}_{2}$ concentration, respectively. Supplemental Figure S1 (https://doi.org/10.3168/jds.2019-16472) indicates that the fluorescence retention of the obtained His-pAT CuNP decreased sharply as the $\mathrm{H}_{2} \mathrm{O}_{2}$ concentration increased from 0 to $12.5 \mu \mathrm{M}$ and then plateaued when the $\mathrm{H}_{2} \mathrm{O}_{2}$ concentration further increased to $50 \mu M$. The inset in Supplemental Figure S1 (https://doi.org/ 10.3168/jds.2019-16472) shows that the change in the fluorescence retention of formed His-pAT CuNP displayed a favorable linearity against the logarithm of $\mathrm{H}_{2} \mathrm{O}_{2}$ concentration ranging from 0.39 to $12.5 \mu M$. The LOD of the formed His-pAT CuNP to the $\mathrm{H}_{2} \mathrm{O}_{2}$ was $0.15 \mu M$, demonstrating the growth of His-pAT CuNP was extremely sensitive to the $\cdot \mathrm{OH}$. We then further investigated the feasibility of using Gox to regulate HispAT CuNP synthesis by mixing His-pAT CuNP growing system with different Gox concentrations under 0.5 $M$ of glucose. Figure 2D shows that the fluorescence retention of formed His-pAT CuNP decreased when Gox concentration increased from 0 to $44.64 \mathrm{fM}$ and then reached a plateau with the further increase in Gox. The fitted linear equation can be described as $y=$ $-19.43 \ln (x)+61.27\left(\mathrm{R}^{2}=0.9803\right)$. The LOD of Gox to the fluorescence retention of His-pAT CuNP was calculated to be $0.23 \mathrm{fM}$. The LOD value was defined as the lowest Gox concentration that generated evident fluorescence retention (90\%) and was approximately 30-fold lower than that of HRP to TMB (Supplemental Figure S2; https://doi.org/10.3168/jds.2019-16472). The above results indicated that an ultralow Gox concentration could produce sufficient amounts of $\mathrm{H}_{2} \mathrm{O}_{2}$ and lead to a remarkable fluorescence signal variation by disrupting the His-pAT CuNP synthesis. These results suggested that the Gox regulating the synthesis of His-pAT CuNP as a signal transducer can improve the sensitivity of sandwich immunoassay for $S$. choleraesuis detection.

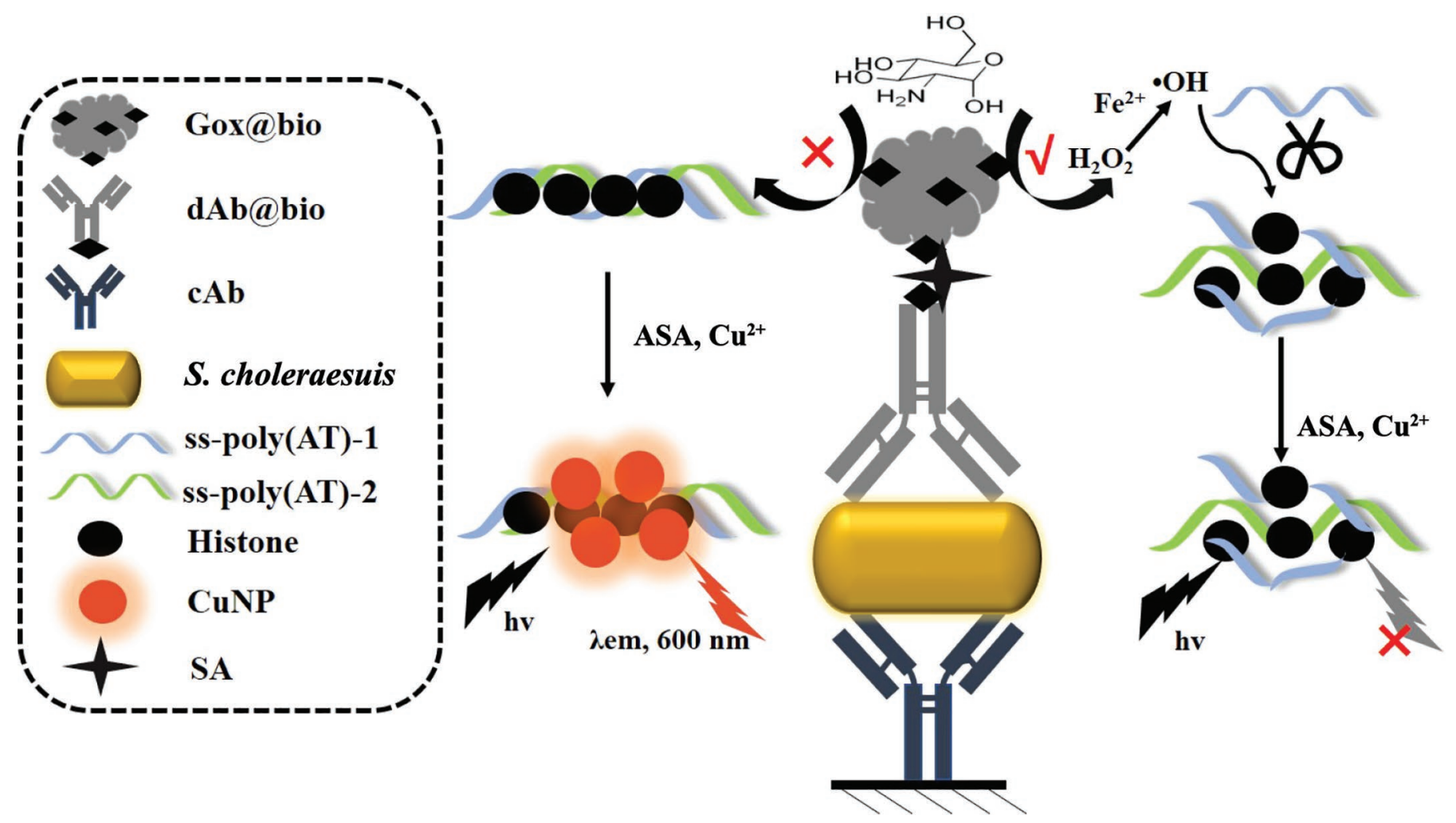

Figure 1. The principle of the proposed fluorescence immunoassay. Gox@bio = biotinylated glucose oxidase; dAb@bio= biotinylated detection antibodies; $\mathrm{cAb}=$ capture antibodies; S. choleraesuis = Samonella choleraesuis; CuNP = copper nanoparticles; $\mathrm{SA}=$ streptavidin; ASA = ascorbic acid; hv $=$ irradiation with light; $\lambda \mathrm{em}=$ wavelength emission. 
Development of Fluorescence Immunoassay Based on His-pAT CuNP as Signal Transducers

To obtain the best performance of sandwich fluorescence immunoassay, we optimized various parameters that can affect detection sensitivity for $S$. choleraesuis, including cAb,dAb@bio, Gox@bio, and SA concentrations. The detailed preparation of dAb@bio and Gox@ bio is shown in the Supplemental Information (https: //doi.org/10.3168/jds.2019-16472). The synthesized dAb@bio and Gox@bio were characterized by adding different concentrations of dAb@bio and Gox@bio (0-4 $\mu \mathrm{g} / \mathrm{mL}$ ) into the SA coated plate wells. Supplemental Figures S3A and 3B (https://doi.org/10.3168/jds.2019 -16472) show that the optical density values increased with increased concentrations of dAb@bio and Gox@ bio, indicating that biotin was successfully coupled on the $\mathrm{dAb}$ and Gox molecules, respectively. The concen-
A

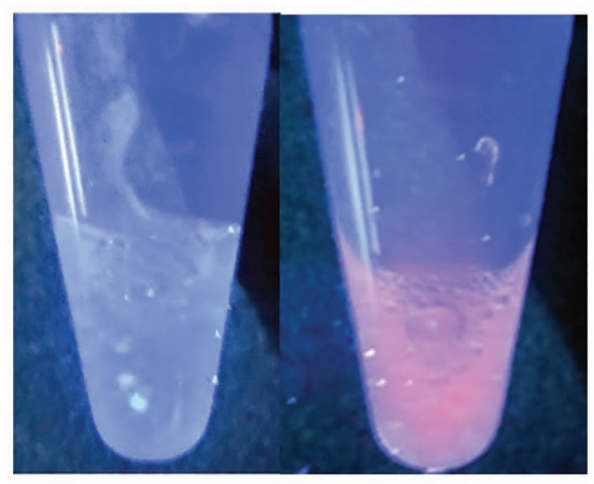

B

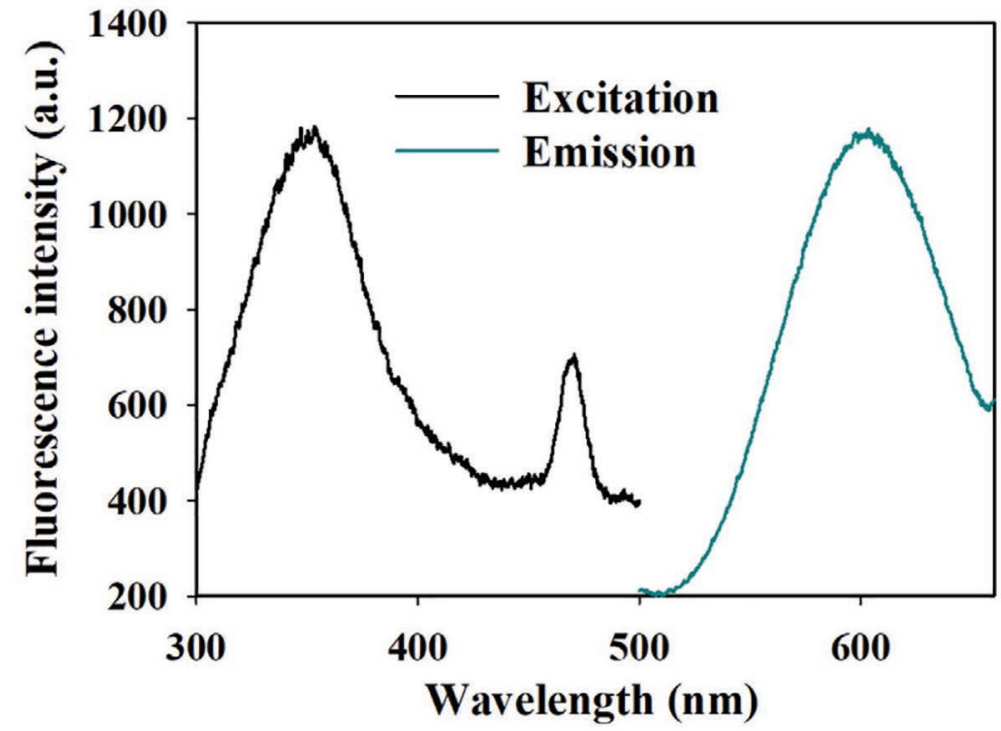

C
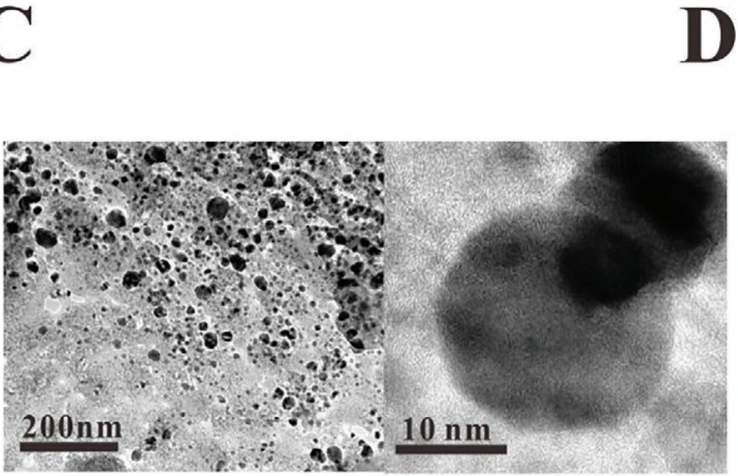

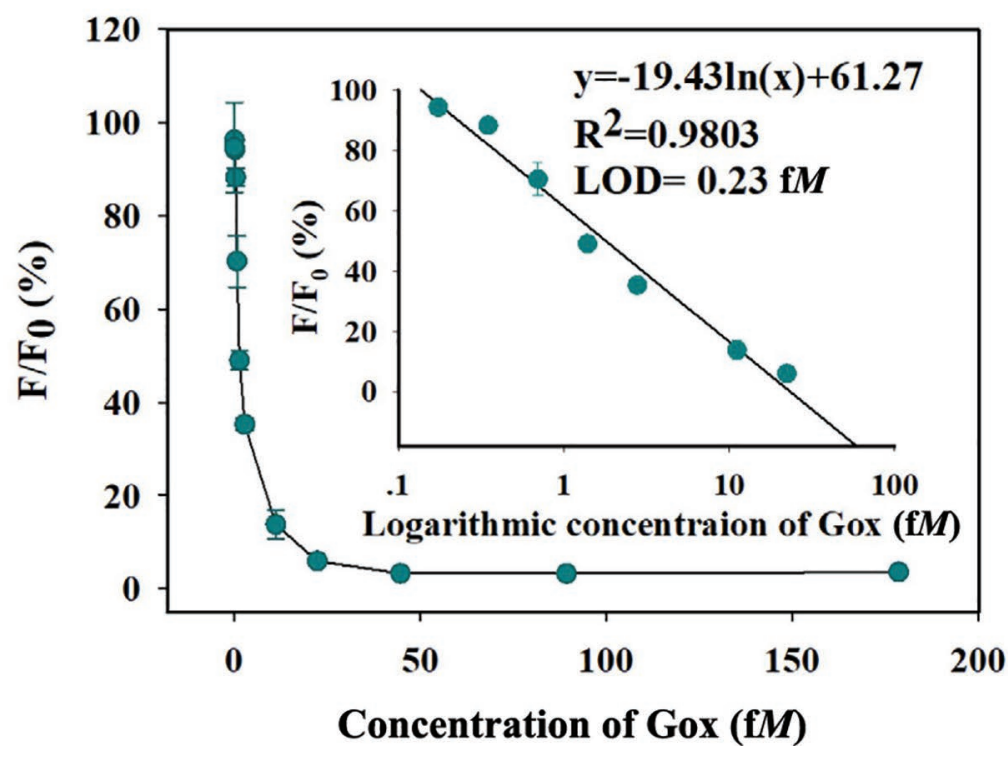

Figure 2. Characterization of histone-ds-poly(AT)-templated copper nanoparticles (His-pAT CuNP). (A) Photographs of His-pAT CuNP solution under UV light (365 nm, left: without $\mathrm{Cu}^{2+}$, right: with $\mathrm{Cu}^{2+}$ ). (B) Excitation and emission spectra of His-pAT CuNP. (C) Left: transmission electron microscopy (TEM) images of His-pAT CuNP; right: high-resolution TEM images of His-pAT CuNP. (D) Sensitivity of glucose oxidase (Gox) toward the His-pAT CuNP synthesis. The Gox was incubated with $0.5 M$ of glucose, $0.5 \mathrm{~m} M$ of Fe ${ }^{2+}$, and $0.63 \mu M$ of ss-poly $(\mathrm{AT})-1$ for $1 \mathrm{~h}$ at $37^{\circ} \mathrm{C}$ before the typical synthesis run of His-pAT CuNP. $\mathrm{F}_{0}$ and $\mathrm{F}$ represented the fluorescence intensities (FI) in the absence and presence of the desired concentration of Gox, respectively. The limit of detection (LOD) of Gox toward the His-pAT CuNP synthesis was defined as the lowest concentration of Gox that FI retained $90 \%$ of $\mathrm{F}_{0}$. The error bars represent the SD of 3 measurements. a.u. $=$ arbitrary units. 
trations of $\mathrm{cAb}$ and $\mathrm{dAb} @$ bio were optimized by the "chessboard titration" method with different $\mathrm{cAb}$ concentrations under a series concentration of dAb@bio on the plate well. The fluorescence retention was used to confirm the optimum parameters. The $S$. choleraesuis concentration for the evaluation of fluorescence retention was set as $8.8 \times 10^{7} \mathrm{cfu} / \mathrm{mL}$. Supplemental Table S1 (https://doi.org/10.3168/jds.2019-16472) shows that the fluorescence retention gradually decreased
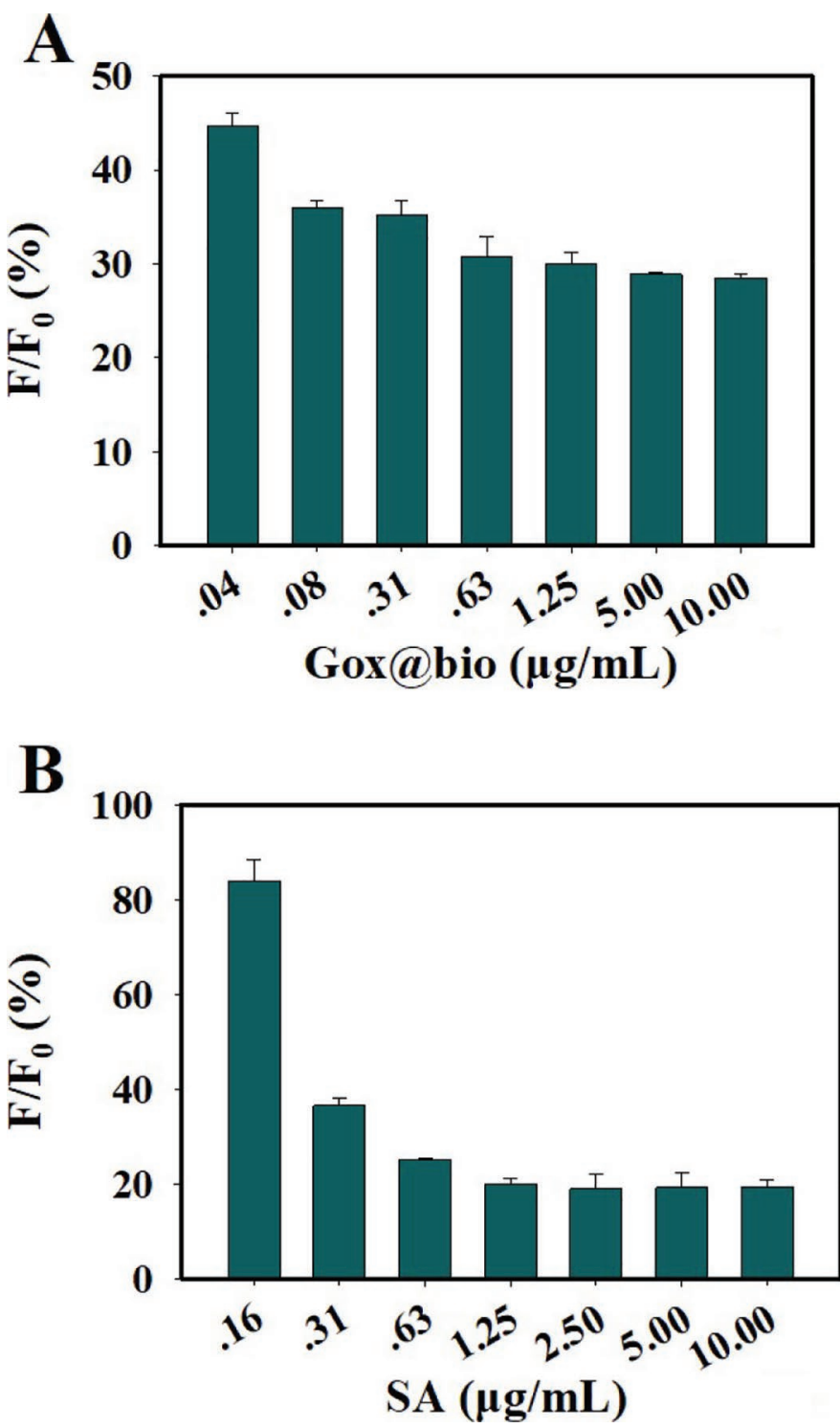

Figure 3. Optimization of the experimental conditions for fluorescence immunoassay. (A) Optimization of biotinylated glucose oxidase (Gox@bio) concentration. The streptavidin (SA) concentration was set as $0.5 \mu \mathrm{g} / \mathrm{mL}$. (B) Optimization of SA concentration. $\mathrm{F}_{0}$ and $\mathrm{F}$ represented the fluorescence intensities (FI) in the absence and presence of $8.8 \times 10^{7} \mathrm{cfu} / \mathrm{mL}$ of Salmonella choleraesuis, respectively. The error bars represent the SD of 3 measurements.
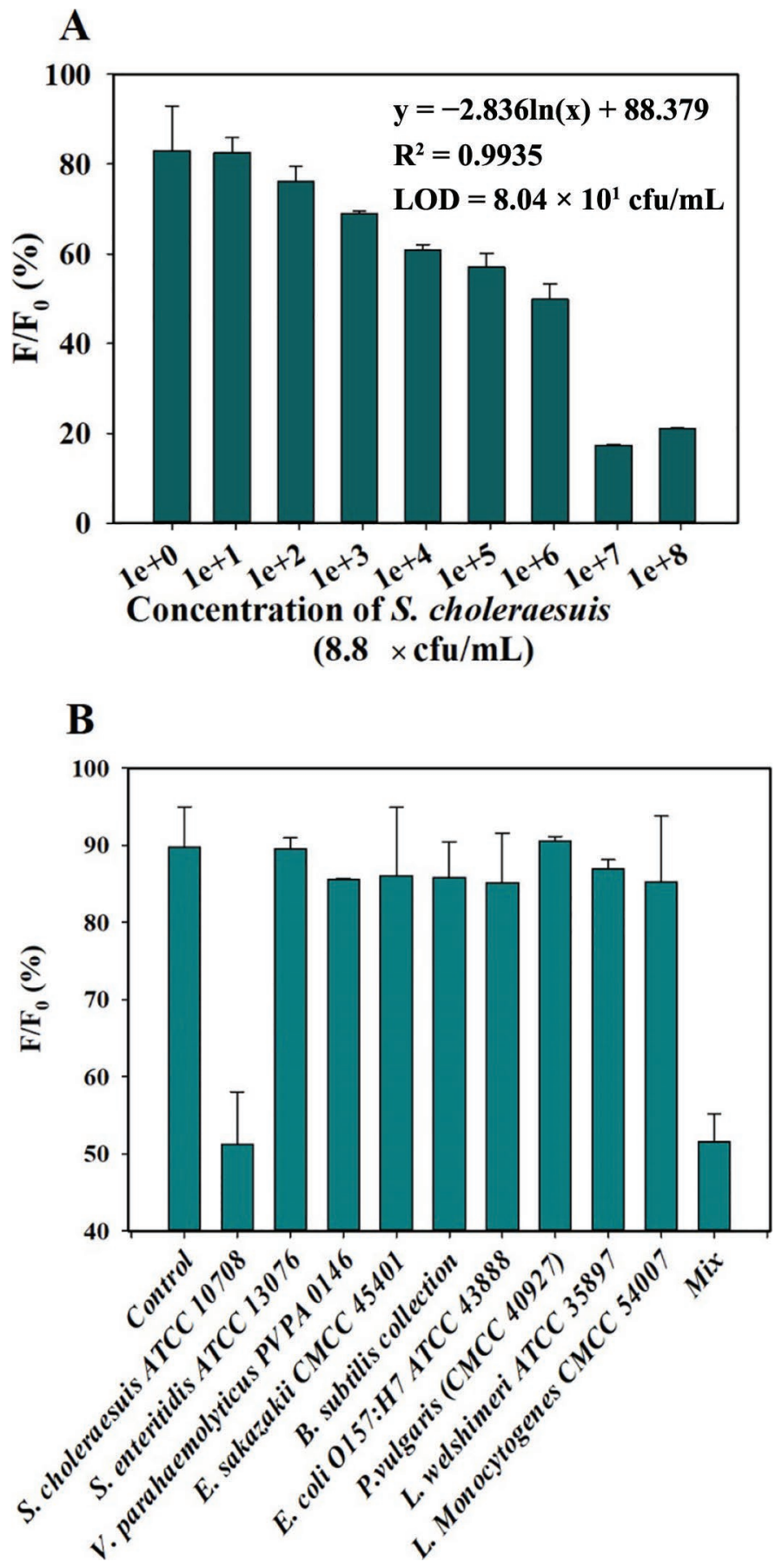

Figure 4. (A) Quantitative assay of Salmonella choleraesuis by using the developed fluorescence immunoassay in PBS solution with $S$. choleraesuis concentration ranging from $8.8 \times 10^{0}$ to $8.8 \times 10^{8} \mathrm{cfu} / \mathrm{mL}$. $\mathrm{F}_{0}$ and $\mathrm{F}$ represented the fluorescence intensities $(\mathrm{FI})$ in the absence and presence of the desired $S$. choleraesuis concentration, respectively. $\mathrm{LOD}=$ limit of detection. (B) Specificity of the developed fluorescence immunoassay. The concentrations of $S$. choleraesuls was at $8.8 \times 10^{6}$ $\mathrm{cfu} / \mathrm{mL}$, whereas other pathogenic bacteria strains were $1 \times 10^{8} \mathrm{cfu} /$ $\mathrm{mL}$. Control is the sterile PBS solution. The error bars represent the $\mathrm{SD}$ of 3 measurements. 
with increased $\mathrm{cAb}$ and $\mathrm{dAb} @$ bio concentration from 0.31 to $2.5 \mu \mathrm{g} / \mathrm{mL}$ and 0.31 to $2.5 \mu \mathrm{g} / \mathrm{mL}$, respectively. Thus, $2.5 \mu \mathrm{g} / \mathrm{mL}$ of $\mathrm{cAb}$ and $\mathrm{dAb} @$ bio was regarded as the optimal concentration. The SA-bio system was used to bridge dAb@bio with Gox@bio. The concentration of Gox@bio and SA were also optimized by determining fluorescence retention for the detection of $S$. choleraesuis at $8.8 \times 10^{7} \mathrm{cfu} / \mathrm{mL}$. Figure $3 \mathrm{~A}$ shows that fluorescence retention decreased from 44.73 to $28.87 \%$ when Gox@bio increased from 0.04 to $5 \mu \mathrm{g} / \mathrm{mL}$ and then plateaued when Gox@bio concentrations further increased. Therefore, $5 \mu \mathrm{g} / \mathrm{mL}$ of Gox@bio was used for subsequent experiments. Figure $3 \mathrm{~B}$ shows that the fluorescence retention decreased from 82 to $18 \%$ with the SA increasing from 0.16 to $1.25 \mu \mathrm{g} / \mathrm{mL}$ and then reached a plateau by further increasing SA concentrations. Thus, $1.25 \mu \mathrm{g} / \mathrm{mL}$ of SA was considered the optimal amount for producing the best detection signal.

Under optimal conditions, the sensitivity of the proposed fluorescence immunoassay was evaluated by detecting different concentrations of $S$. choleraesuis (from $8.8 \times 10^{0}$ to $\left.8.8 \times 10^{8} \mathrm{cfu} / \mathrm{mL}\right)$. The calibration curve was constructed by plotting the fluorescence retention with $\mathrm{F} / \mathrm{F}_{0} \times 100 \%$ values against the logarithmic concentration of $S$. choleraesuis. As presented in Figure $4 \mathrm{~A}$, the regression equation of the proposed method for quantitative $S$. choleraesuis detection can be described as $y=-2.836 \ln (x)+88.379\left(\mathrm{R}^{2}=0.9935\right)$, with a good linear detection ranging from $8.8 \times 10^{1}$ to $8.8 \times 10^{6}$. In the equation, $y$ is fluorescence retention, and $x$ is $S$. choleraesuis concentration. The error bars were based on triplicate measurements. The LOD was calculated as $8.04 \times 10^{1} \mathrm{cfu} / \mathrm{mL}$ in accordance with the mean of the determined fluorescence retention of blank samples plus a 3-fold standard deviation. This value is 1,000 times lower than that of the conventional TMB-based ELISA $\left(8.7 \times 10^{4} \mathrm{cfu} / \mathrm{mL}\right.$, Supplemental Figure S4; https:// doi.org/10.3168/jds.2019-16472). Compared with other recently developed methods for Salmonella detection, the proposed method is significantly better and slightly lower than that of quantum dot-based fluorescence and electrochemical response-based immunoassays, respectively (Table 1).

Specificity was determined by evaluating the crossreaction of the proposed fluorescence immunoassay with $S$. choleraesuis, S. enteritidis, and other common pathogenic bacterial strains, including E. coli O157:H7, L. monocytogenes, L. welshimeri, E. sakazakii, V. parahaemolyticus, P. vulgaris, and B. subtilis. Sterile PBS was used as the negative control. Compared with the negative control, a lower fluorescence retention was observed when the $S$. choleraesuis concentration was $8.8 \times 10^{6} \mathrm{cfu} / \mathrm{mL}$, whereas no significant differences of fluorescence retention were observed when other nontarget bacteria were detected at concentrations of $1 \times$ $10^{8} \mathrm{cfu} / \mathrm{mL}$ (Figure $4 \mathrm{~B}$ ). These findings demonstrate the high selectivity of the proposed fluorescence immunoassay for $S$. choleraesuis.

The accuracy and precision of our proposed method were evaluated by analyzing 4 different concentrations $\left(8.8 \times 10^{1}, 8.8 \times 10^{2}, 8.8 \times 10^{3}\right.$, and $\left.8.8 \times 10^{4} \mathrm{cfu} / \mathrm{mL}\right)$ of S. choleraesuis-spiked pasteurized whole milk samples. The effects of sample matrix were removed through the 2-step large-volume immunomagnetic separation method developed by our group for the isolation and enrichment of $S$. choleraesuis from milk samples before fluorescence analysis. Table 2 shows that average recoveries for intra- and inter-assay ranged from 73.52 to $96.59 \%$ and 66.99 to $98.24 \%$ with a coefficient of variation ranging from 6.85 to $31.26 \%$ and 5.46 to $17.99 \%$, respectively. These results signified that the proposed fluorescence immunoassay method can be used for detection of $S$. choleraseuis in real milk samples.

\section{CONCLUSIONS}

We demonstrated that His-pAT CuNP can be used as signal outputs of immunoassay for the sensitive de-

Table 1. Comparison of the sensitivity of different methods for Salmonella detection

\begin{tabular}{|c|c|c|c|c|}
\hline Method & Signal output & $\begin{array}{l}\text { Linear range } \\
\quad(\mathrm{cfu} / \mathrm{mL})\end{array}$ & $\begin{array}{c}\mathrm{LOD}^{1} \\
(\mathrm{cfu} / \mathrm{mL})\end{array}$ & Reference \\
\hline Fluorescence immunoassay & $\begin{array}{l}\text { Quantum dot-based fluorescence } \\
\text { response }\end{array}$ & $2.50 \times 10^{3}$ to $1.95 \times 10^{8}$ & 500 & Kuang et al., 2013 \\
\hline Microfluidic immunoassay & $\begin{array}{l}\text { Quantum dot-based fluorescence } \\
\text { response }\end{array}$ & 0 to $10^{6}$ & 1,000 & Kim et al., 2015 \\
\hline Microfluidic immunoassay & Electrochemical response & $10^{1}$ to $10^{7}$ & 1.56 & Singh et al., 2018 \\
\hline Fluorescence immunoassay & $\begin{array}{l}\text { Copper nanoparticle-based } \\
\text { fluorescence response }\end{array}$ & $8.8 \times 10^{1}$ to $8.8 \times 10^{6}$ & 80.4 & This work \\
\hline
\end{tabular}

${ }^{1} \mathrm{LOD}=$ limit of detection. 
Table 2. Recovery and precision of the proposed fluorescence immunoassay

\begin{tabular}{|c|c|c|c|}
\hline Salmonella choleraesuis added (cfu/mL) & $\begin{array}{c}\text { S. choleraesuis } \\
\text { detected }(\mathrm{cfu} / \mathrm{mL})\end{array}$ & Recovery (\%) & $\operatorname{RSD}^{1}(\%)$ \\
\hline \multicolumn{4}{|c|}{ Proposed fluorescence immunoassay, intra-day assay $(n=3)$} \\
\hline $8.8 \times 10^{1}$ & $6.47 \times 10^{1}$ & 73.52 & 20.56 \\
\hline $8.8 \times 10^{3}$ & $7.09 \times 10^{3}$ & 80.57 & 6.85 \\
\hline $8.8 \times 10^{4}$ & $8.50 \times 10^{4}$ & 96.59 & 9.06 \\
\hline \multicolumn{4}{|c|}{ Proposed fluorescence immunoassay, inter-day assay $(n=3)$} \\
\hline $8.8 \times 10^{1}$ & $5.90 \times 10^{1}$ & 66.99 & 9.75 \\
\hline $8.8 \times 10^{4}$ & $8.06 \times 10^{4}$ & 91.59 & 5.46 \\
\hline
\end{tabular}

${ }^{1} \mathrm{RSD}=$ relative standard deviation.

tection of foodborne pathogens. Given the ultra-high sensitivity of His-pAT CuNP synthesis toward $\cdot \mathrm{OH}$, the proposed fluorescence immunoassay exhibited outstanding sensitivity for the detection of $S$. choleraesuis, having an LOD of $8.04 \times 10^{1} \mathrm{cfu} / \mathrm{mL}$. This result is 3 orders of magnitude lower than that obtained by TMBbased traditional immunoassay. The proposed immunoassay showed excellent specificity, good precision, and suitable practicability, and His-pAT CuNP exhibited good stable fluorescence signal in the whole process of immunoassay. In brief, our developed fluorescence immunoassay provided a new potential for developing rapid and highly sensitive detection in food safety control, environment monitoring, and clinical diagnosis.

\section{ACKNOWLEDGMENTS}

This work was supported by a grant from the National Key Research and Development Program of China (2018YFC1602202 and 2018YFC1602203), National Natural Science Foundation of China (31760485), National Agricultural Science and Technology Innovation Program, and Student's Platform for Innovation and Entrepreneurship Training Program from Nanchang University (201802187). Ying Xiong acknowledges financial support from the Chinese Scholarship Council.

\section{REFERENCES}

Abdalhai, M. H., A. M. Fernandes, X. Xia, A. Musa, J. Ji, and X Sun. 2015. Electrochemical genosensor to detect pathogenic bacteria (Escherichia coli O157: H7) as applied in real food samples (fresh beef) to improve food safety and quality control. J. Agric. Food Chem. 63:5017-5025.

Abdalhai, M. H., A. N. Maximiano Fernandes, M. Bashari, J. Ji, Q. He, and X. Sun. 2014. Rapid and sensitive detection of foodborne pathogenic bacteria (Staphylococcus aureus) using an electrochemical DNA genomic biosensor and its application in fresh beef. J. Agric. Food Chem. 62:12659-12667.

CDC. (The Centers for Disease Control and Prevention). 2018. Estimates of foodborne illness in the United States. Accessed Nov. 5 , 2018. https://www.cdc.gov/foodborneburden/index.html.
Chakraborty, S., S. Babanova, R. C. Rocha, A. Desireddy, K. Artyushkova, A. E. Boncella, P. Atanassov, and J. S. Martinez. 2015. A hybrid DNA-templated gold nanocluster for enhanced enzymatic reduction of oxygen. J. Am. Chem. Soc. 137:11678-11687.

Chen, R., X. Huang, J. Li, S. Shan, W. Lai, and Y. Xiong. 2016. A novel fluorescence immunoassay for the sensitive detection of Escherichia coli O157: H7 in milk based on catalase-mediated fluorescence quenching of CdTe quantum dots. Anal. Chim. Acta 947:50-57.

Chen, R., X. Huang, H. Xu, Y. Xiong, and Y. Li. 2015. Plasmonic enzyme-linked immunosorbent assay using nanospherical brushes as a catalase container for colorimetric detection of ultralow concentrations of Listeria monocytogenes. ACS Appl. Mater. Interfaces 7:28632-28639.

Hiitiö, H., S. Pyörälä, S. Taponen, P. Rajala-Schultz, and H. Simojoki. 2018. Elimination of experimentally induced bovine intramammary infection assessed by multiplex real-time PCR and bacterial culture. J. Dairy Sci. 101:5267-5276.

Kim, G., J. H. Moon, C. Y. Moh, and J. G. Lim. 2015. A microfluidic nano-biosensor for the detection of pathogenic Salmonella. Biosens. Bioelectron. 67:243-247.

Kuang, H., G. Cui, X. Chen, H. Yin, Q. Yong, L. Xu, C. Peng, L. Wang, and C. Xu. 2013. A one-step homogeneous sandwich immunosensor for Salmonella detection based on magnetic nanoparticles (MNPs) and quantum dots (QDs). Int. J. Mol. Sci. 14:8603-8610.

Lian, J., Q. Liu, Y. Jin, and B. Li. 2017. Histone-DNA interaction: An effective approach to improve the fluorescence intensity and stability of DNA-templated $\mathrm{Cu}$ nanoclusters. Chem. Commun. (Camb.) $53: 12568-12571$.

Liu, J. 2014. DNA-stabilized, fluorescent, metal nanoclusters for biosensor development. TrAC Trends in Analytical Chemistry 58:99111.

Luo, D., X. Huang, Y. Mao, C. Chen, F. Li, H. Xu, and Y. Xiong. 2017. Two-step large-volume magnetic separation combined with PCR assay for sensitive detection of Listeria monocytogenes in pasteurized milk. J. Dairy Sci. 100:7883-7890.

Luo, K., H.-Y. Kim, M.-H. Oh, and Y.-R. Kim. 2018. Based lateral flow strip assay for the detection of foodborne pathogens: Principles, applications, technological challenges and opportunities. Crit. Rev. Food Sci. Nutr. 12:1-14.

Ma, X., Y. Jiang, F. Jia, Y. Yu, J. Chen, and Z. Wang. 2014. An aptamer-based electrochemical biosensor for the detection of Salmonella. J. Microbiol. Methods 98:94-98.

Mao, Z., Z. Qing, T. Qing, F. Xu, L. Wen, X. He, D. He, H. Shi, and K. Wang. 2015. Poly (thymine)-templated copper nanoparticles as a fluorescent indicator for hydrogen peroxide and oxidase-based biosensing. Anal. Chem. 87:7454-7460.

Niyomdecha, S., W. Limbut, A. Numnuam, P. Kanatharana, R. Charlermroj, N. Karoonuthaisiri, and P. Thavarungkul. 2018. Phagebased capacitive biosensor for Salmonella detection. Talanta 188:658-664. 
Petty, J. T., J. Zheng, N. V. Hud, and R. M. Dickson. 2004. DNA-templated Ag nanocluster formation. J. Am. Chem. Soc. 126:52075212.

Qing, Z., X. He, T. Qing, K. Wang, H. Shi, D. He, Z. Zou, L. Yan, F. Xu, and X. Ye. 2013. Poly (thymine)-templated fluorescent copper nanoparticles for ultrasensitive label-free nuclease assay and its inhibitors screening. Anal. Chem. 85:12138-12143.

Ranjbar, S., S. Shahrokhian, and F. Nurmohammadi. 2018. Nanoporous gold as a suitable substrate for preparation of a new sensitive electrochemical aptasensor for detection of Salmonella typhimuri$u m$. Sens. Actuators B Chem. 255:1536-1544.

Shan, S., W. Lai, Y. Xiong, H. Wei, and H. Xu. 2015. Novel strategies to enhance lateral flow immunoassay sensitivity for detecting foodborne pathogens. J. Agric. Food Chem. 63:745-753.

Shang, Y., J. Sun, Y. Ye, J. Zhang, Y. Zhang, and X. Sun. 2018. Loop-mediated isothermal amplification-based microfluidic chip for pathogen detection. Crit. Rev. Food Sci. Nutr. 20:1-24.
Singh, C., M. A. Ali, V. Kumar, R. Ahmad, and G. Sumana. 2018. Functionalized $\mathrm{MoS}_{2}$ nanosheets assembled microfluidic immunosensor for highly sensitive detection of food pathogen. Sens. Actuators B Chem. 259:1090-1098.

Tang, Y., F. Feng, F. He, S. Wang, Y. Li, and D. Zhu. 2006. Direct visualization of enzymatic cleavage and oxidative damage by hydroxyl radicals of single-stranded DNA with a cationic polythiophene derivative. J. Am. Chem. Soc. 128:14972-14976.

World Health Organization. 2011. Foodborne Disease Strategy: 2010 update. United Kingdom. Accessed May 2011. http://multimedia food.gov.uk/multimedia/pdfs/fds2015.pdf.

Xiong, Y., B. Gao, K. Wu, Y. Wu, Y. Chai, X. Huang, and Y. Xiong. 2018. Fluorescence immunoassay based on the enzyme cleaving ssDNA to regulate the synthesis of histone-ds-poly (AT) templated copper nanoparticles. Nanoscale 10:19890-19897. 